\title{
Human-Mobility-Based Spray and Wait: Efficient Routing Protocol for Pocket Switched Networks
}

\author{
Namita Mehta ${ }^{1}$ and Mehul Shah ${ }^{2}$ \\ ${ }^{1}$ Faculty of Electronics \& Communication Engg. \\ $B \& B$ Institute of Technology, V.V.Nagar \\ ${ }^{2}$ Associate Professor, Department of Communication Engg. \\ G.H.Patel College of Engineering \& Technology, V.V.Nagar \\ Gujrat, India. \\ uvmehta2000@yahoo.com, \\ mehul_gcet@yahoo.com
}

\begin{abstract}
Delay and/or Disruption-Tolerant Networking (DTN) is a novel communication prototype that can span across multiple networks and deal with unpredicted conditions in the Internet model. Pocket Switched Networks (PSN) are delay tolerant networks with hand held devices. Nodes in a PSN make use of a store-carry-and-forward paradigm to communicate along with themselves. The routing protocols performances that have been developed for DTN based on the movement pattern of the nodes. We propose a Human Mobility based Spray \& Wait Routing protocol (HMSaW). This protocol makes use of truncated power law distribution of pause time and flight length of human mobility to smartly forward data in DTN and PSN. Simulation results show that the proposed HMSaW shows better delivery ratio and the communication overhead, with marginal reduction in average delay compared to existing PROPHET and Spray \& Wait Routing protocol.
\end{abstract}

Keywords: Pocket Switched Network, Delay Tolerant Networks, Routing Protocols, Human Mobility, TLW model

\section{Introduction}

Usually, in traditional networks, like the Internet, there exists a fully connected path among any pair of nodes at any time. However, in Delay Tolerant Networks (DTN) nodes may be partitioned into two disconnected subsets when directly connected path is supposed to be broken as the movement or failure of some nodes. Hence, the data transmission requirements cannot be satisfied by traditional routing networks/schemes. Hence the critical and challenging issue for DTN is how to efficiently route data. DTN guarantee to facilitate communication between "challenged" networks [1,2], which covers in space networks, under-water networks, military networks ,sensor networks, mobile adhoc networks, vehicular networks, inter-planetary networks [3], and low cost networks. The basic scheme of networks is to reach information of any form, from source to sink/destination. For any given time interval, in DTN wireless network, the inter connectivity of the network is sustained by each other nodes when they encounter within transmission ranges. A message inside the buffer of nodes stores until an appropriate communication opportunity arises. Pocket Switched Networks (PSN) [9] scenario is similar to DTN, where data has to be communicated with hand held devices such as cell phones.

The rest of the paper is organized as follows. Section 2 contains popular routing protocols in DTN; Section 3 for System model, Section 4 and 5 provides information of 
proposed novel protocol algorithm, simulation done in Section 6 and draws a conclusion in Section 7.

\section{Routing Protocol in DTN}

In DTN, due to high delay and intermittent disconnection in these networks, it won't assure that fully connected path exist between source and destination. A number of solutions have been proposed to tackle in routing of DTNs. Routing protocols plays a major role to deliver messages between two communicating nodes. Epidemic Routing [4] is the routing schemes, used the store, carry and forward paradigm. Although, Epidemic Routing (ER) accomplish delivery of $100 \%$ messages, but it has limitation not to reduce replication. Also achieve high delivery rate at the cost of the network resource utilization, such as occupy buffer space and transmission bandwidth. But in congested traffic network, performance of ER (Epidemic Routing) will get worse. Hence to reduce the overhead of flooding and improve ER's performance, numerous schemes have been projected based on routing.

\subsection{Prophet}

PROPHET, Probabilistic Routing Protocol using History of Encounters and Transitivity, is an evolution of Epidemic Routing that introduced the concept of delivery predictability [5].The basic assumption in the PROPHET is that mobility of nodes is not entirely in random manner, but it has a number of deterministic properties e.g. repeating behavior. It means in PROPHET it might be possible that node has visited a location several time. Due to this possibility, this pattern will be repeated in the future. In PROPHET every node uses probabilistic metric called delivery predictability to transfer messages to a reliable node. The higher delivery predictability for a node indicates that it is more reliable than other nodes to forward message to destination. The basic difference of Prophet than Epidemic Routing is its forwarding strategy. When two nodes meet, Prophet allows transfer of a message to the other node only if the delivery predictability of the destination of the message is higher at the other node.

\subsection{Spray and Wait}

In Spray and wait [6], the speed of Epidemic Routing controlled by controlling the number of packet copies in spray phase and utilizing direct transmission in wait phase with the simplicity and reliability of direct transmission. The Spray and Wait process consists of two phases, namely, the spray phase and other, the wait phase. In the spray phase, a node generates L copies of the packets which spread it to relay nodes. At any instant, when a node encounters the other node within transmission range, the node checks $L$ copies. If $L$ has the value higher than 1 , then the node sprays half (i.e. L / 2) copies of the packets to its neighbor and update $L$. These will continue till only single copy left. A wait phase starts when there is only single copy left. The direct transmission is used to send this single copy to the destination directly. Each $\mathrm{N}$ node carries a message copy to perform direct message transmission until delivered the message to the destination successfully.

\section{System Model}

Human mobility has a significant influence on the performance of networked systems that involve daily human activities. Therefore, studying and finding fundamental characteristics of human mobility and developing realistic human mobility models are essential for optimum construction of these systems.

Work based on various real world traces and accomplished that the movement of real people from various outdoor settings follows a Levy Walk [7]. People move within a 
predefined (also confined) area for routine activities. These tendencies are not confined in common mobility models such as random way point (RWP). A performance study of routing protocolsTLW mobility model brings out in this article. The mobile users having distinctiveness human walk in this model. Human walk characteristics followed by flight length, pause time and inter-contact time. These characteristics illustrate in Figure 1.

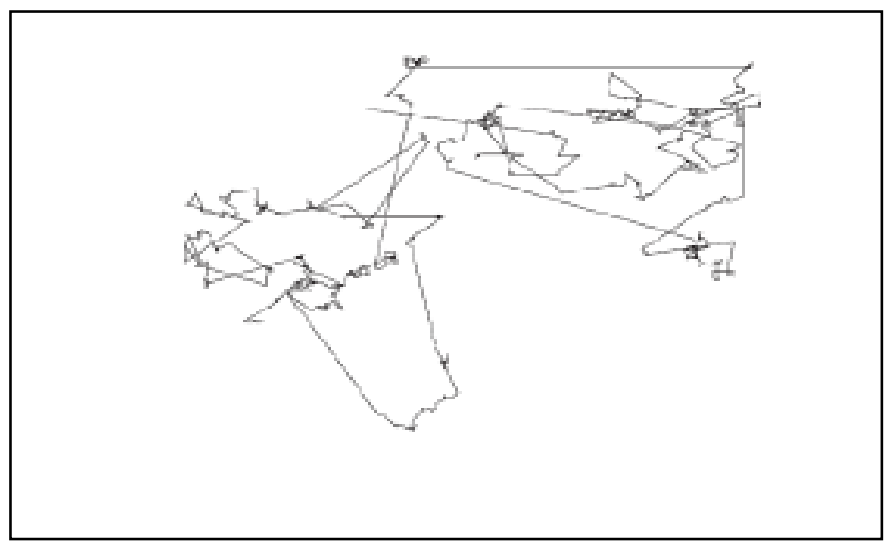

Figure 1. Waypoints For Levy Walk Mobility Model

a) Flight length probability for Levy walk model is $\rho(l) \alpha|l|^{-(1+\alpha)} ; 0<\alpha \leq 2$

Here lmax known as the maximum flight length

b) Pause time Probability for Levy walk model is

$$
\phi(t) \alpha t^{-(1+\beta)} ; 0<\beta \leq 2
$$

c) A uniform distribution of tuning angles within $[0,360]$

d) Velocity increases as flight lengths increase.

\section{Design of Human-Mobility-Based Spray and Wait Routing Algorithm}

Usual Spray and Wait routing, in spray phase, for every message originating from a source node, L copies of packets are initially spread which receives possibly a copy by other nodes to L distinct "relays". In an optimal Spray and Wait scheme namely, Binary Spray and Wait (BSW), is proposed in [6] to get faster the spray phase which improve the routing performance. As when any node has packets $\mathrm{L}$ more than 1 copies, half of the copies hands over to its encountered node until $\mathrm{L}=1$. On other side, if the relay is a very inactive node, which might have by no means in contacted with other neighbor nodes, handing over half the copies to such a node. This means that half copies will not be transmitted to the destination which results probability for half of the relay will be exhausted. In order to tackle this problem, we establish a novel scheme in the spray phase. In this scenario, we have calculated a relay weight to the destinations for every node and exchange different numbers of copies to the nodes based on their delivery probabilities or relay weight.

Two human walk context metrics have been added to modify the Spray and Wait Routing protocol called Flight length parameter $\left(H_{u}\right)$ and Pause time parameter $\left(P_{u}\right)$.

\subsection{Human Walk Context Prameter}

\subsubsection{Flight length parameter $H_{u}$ :}

The parameter $\mathrm{H}_{\mathrm{u}}$ is calculated at each node based on the decision whether a given flight length should be considered as a super flight length or not. The determination of 
parameter $\mathrm{H}_{\mathrm{u}}$ depends on calculation of flight, which a node takes, it is a super flight length or not [8].

a) Super Flight Length : A mobile nodes having set U, follows the flight length distribution as a truncated power law. For all its subset of nodes $u$, a value $\zeta$ exists. Now if a value of flight length is higher than $\zeta$, all nodes of such a subset again pass through that flight length with higher probability. For any node u,the flight length above $\zeta$ can be identified by $S_{\mathrm{f}(\mathrm{u})}$ and characterized by Super flight length. $\mathrm{S}_{\operatorname{len}(\mathrm{u})}$ signify the definite physical distance corresponds to Super flight length.

$$
S_{l e n(u)}=\alpha^{\prime} S_{f(u)}(1)
$$

Where $\alpha^{\prime}$ is a constant which depends on environmental conditions and mobility location. In usual practice, $S_{\text {count } u \text { }}$ describes in (2) is incremented every time, when flight length of a node make more than $S_{\operatorname{len}(u)}$ as the characteristics of human walk.

$$
\begin{aligned}
& \left.\mathrm{H}_{(\mathrm{u})}=\frac{1}{1+e^{-2(\operatorname{Scount}(u)-1)}}\right\} ; \mathrm{S}_{\operatorname{count}(\mathrm{u})}>0 \\
& \mathrm{H}_{(\mathrm{u})}=0, \quad ; \mathrm{S}_{\text {count(u) }}=0(2)
\end{aligned}
$$

\subsubsection{Pause Time Parameter $P_{u}$ :}

The Pause time parameter $\mathrm{P}_{\mathrm{u}}$ calculated based on whether a given pause is a super pause or not. Hence a super pause time will be defined as below.

a) Super Pause Time : A mobile nodes, having set U, follows the pause time distribution as a truncated power law. For all its subset of nodes $u$, a value $\xi$ exists. If the value of pause time is higher than $\xi$, we call as super pause $\mathrm{P}_{\mathrm{s}(\mathrm{u})}$ of node $\mathrm{u}$. The $\mathrm{P}_{\text {stime(u) }}$ denote actual time relates to super pause time. Then,

$$
P_{s t i m e(u)}=\beta^{\prime} P_{s(u)}
$$

Where $\beta$ ' is a constant. Super pause counter $P_{\text {scount }(u)}$ in (4) is changed from 1 to 0 if a node pauses for more than $P_{\text {stime }(u)}$ time.[8]

$$
\begin{gathered}
P_{(u)}=0 \quad ; P_{\text {scount }(u)}>0, \\
P_{(u)}=1 \quad ; P_{\text {scount }(u)}<0,
\end{gathered}
$$

We have modified the Spray and Wait protocol by calculation of relay weight for every destination node. Based on their delivery probabilities, exchange different numbers of copies to the nodes.

\section{Proposed HMSaW Algorithm}

The HMSaW algorithm is proposed and implemented in Delay Tolerant Network to accomplish high delivery ratio with lower delivery delay. Proposed HMSaW algorithm divided in to two phases namely spray phase and wait phase.

\subsection{Spray Phase}

a) Message is originated from source node and initially fixed (L) numbers of copies are generated.

b) At the beginning of data collection round, each node finds all its neighbors within defined transmission range. Then each node broadcast $\mathrm{NBR}_{\text {dis }}$ (Neighbor Discovery) message periodically having its own ID. 
c) After a predefined time, each node receives $\mathrm{NBR}_{\text {dis }}$ message from its neighbors. Based on $\mathrm{NBR}_{\text {dis }}$ message, each node now calculate the change degree of connectivity by $\mathrm{Cd}_{\mathrm{cu}}$ by

$$
\begin{aligned}
& \left|\mathrm{Nu}_{\mathrm{t}-1} \mathrm{UNu_{ \textrm {t } }}\right|-\left|\mathrm{Nu}_{\mathrm{t}-1} \cap \mathrm{Nu}_{\mathrm{t}}\right| \\
& \mathrm{Cd}_{\mathrm{c}(\mathrm{u})}= \\
& \left|\mathrm{Nu}_{\mathrm{t}-1} \mathrm{U} \mathrm{Nu} \mathrm{Nu}_{\mathrm{t}}\right|
\end{aligned}
$$

where $\mathrm{N}_{u t}$ is the number of neighbor nodes $u$ arrive within transmission range at time $t$ and $\mathrm{N}_{u t-1}$ is the number of neighbor nodes arrive within range at time $t-1$.The $\mathrm{Cd}_{c u}$ constraint relates with the number of nodes that have transitioned from in-reach to out-reach status or vice versa in the time interval $[t-1, t]$.

d) Each node, calculates the battery parameter $\mathrm{Bat}_{\mathrm{u}}$. If battery parameter has value 1 which means full battery and value 0 means an empty battery.

e) Each node finds $H_{u}$ as per (2).

f) Each node finds $P_{u}$ as per (4).

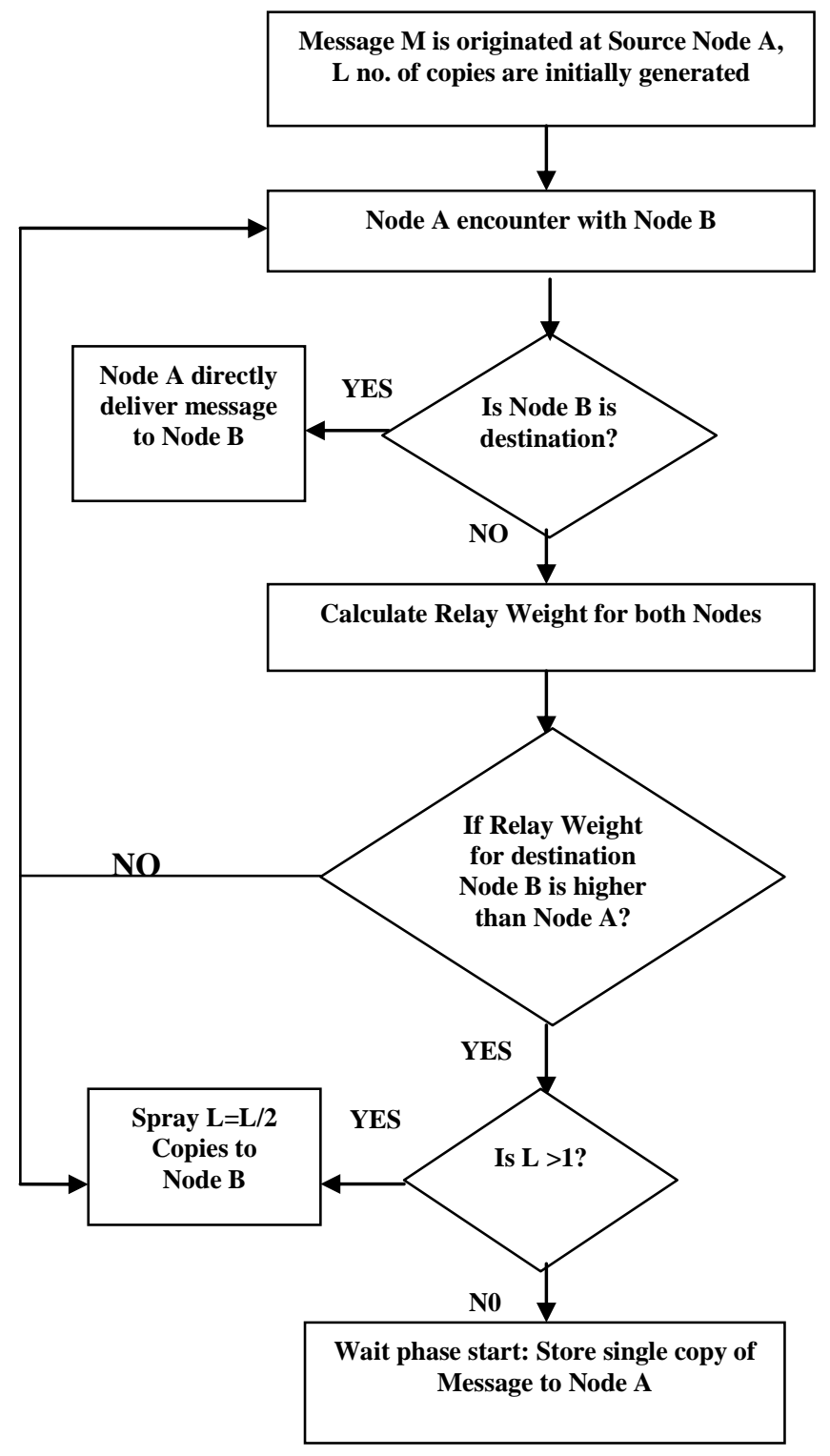

Figure 2. Flowchart of Proposed Hmsaw 
g) All nodes calculate the Relay Weight parameter $\mathrm{W}_{\mathrm{u}}$.

$$
\mathrm{W}_{\mathrm{u}}=\mathrm{W}_{1} \mathrm{Cdc}_{\mathrm{u}}+\mathrm{W}_{2} \mathrm{Bat}_{\mathrm{u}}+\mathrm{W}_{3} H_{u}+\mathrm{W}_{4} P_{u}
$$

Where $\mathrm{W}_{1}, \mathrm{~W}_{2}, \mathrm{~W}_{3}$ and $\mathrm{W}_{4}$ are randomly chosen weighting factors which satisfying $\Sigma_{\mathrm{i}=1}^{4} \mathrm{~W}_{\mathrm{i}}=1$. As the value of weight parameter $\left(\mathrm{W}_{\mathrm{u}}\right)$ higher means, nodes are more feasible and will be a relay node.

h) Data Transmission:

Each message having its unique message ID. Message will be transferred to preferred relay node with highest weight value and nodes are available within transmission range else stored message in its buffer.

\section{i) Data Reception}

When a relay node receives data, process and updates its routing table in case of routing message. But if it is data message, acknowledge (ACK) sent, stored in its buffer and utilize the message. Messages are deleted only after receiving ACK by some relay node or buffer becomes full.

\subsection{Wait Phase}

The wait phase of HMSaW process is analogous to wait phase of Spray and Wait routing protocol. When only single copy left, a wait phase start and direct transmission routing is used to forward single copy from relay node to the destination directly.

\section{Simulation and Analysis}

\subsection{Simulation Parameter}

The performance and evaluation of the novel routing by making variation in the Spray and Wait routing using MATLAB simulator. The main simulation environment parameters are listed in Table 1.

Table 1. Simulation Network Parameter

\begin{tabular}{|c|l|l|}
\hline Protocol & Parameter & Value \\
\hline & Simulation Area & $1000 \mathrm{~m} \mathrm{X} \mathrm{1000} \mathrm{m}$ \\
\hline ALL & Simulation Time & 60 minutes \\
\hline & Mobility Model & TLW \\
\hline & Buffer Size[bytes] & $100,300,450,600,750,900$ \\
\hline & Node & 50 \\
\hline Prophet & $\begin{array}{c}\text { Transmission } \\
\text { Range }\end{array}$ & 50 \\
\hline & Updating factor,$\alpha$ & 0.75 \\
\hline Transivity constant & 0.25 \\
\hline HMSaW & Aging constant,$\gamma$ & 0.98 \\
\hline HMSaW & $\begin{array}{c}\text { Initial number of } \\
\text { copies }\end{array}$ & 10 \\
\hline & $\begin{array}{l}\text { Weight Parameter } \\
\text { (W1,W2,W3,W4) }\end{array}$ & {$[0.4,0.2,0.2,0.2]$} \\
\hline
\end{tabular}

\subsection{Metrics used to Measure the Performance}

There are three metrics are used to measure the performance of the different protocols: 
- Delivery Ratio: The delivery ratio is the ratio of total number of messages delivered to their destination to total number of created messages at source node.

$$
\text { Delivery Ratio }=\frac{\text { Total Delivered Message }}{\text { Total Generated Message }}
$$

- Overhead Ratio: The overhead ratio reflects how many redundant packets are relayed to deliver one packet. It simply reflects transmission cost in a network.

$$
\text { Overhead }=\frac{\text { Total Relayed Message }- \text { Total Delivered Message }}{\text { Total Generated Message }}
$$

- Average delay: The average delay is the time between messages is created and the messages are received at a destination.

\subsection{Simulation Results}

\subsubsection{Impact of Varying Buffer Size}

Evaluate the performance of the novel routing compare with existing routing protocols, like Spray and Wait and Prophet routing. The performance can be compared with various parameters i.e. the delivery ratio, average delay and communication overhead under different buffer sizes. Simulation results for above parameters are shown in Figure 2, Figure 3 and Figure 4.

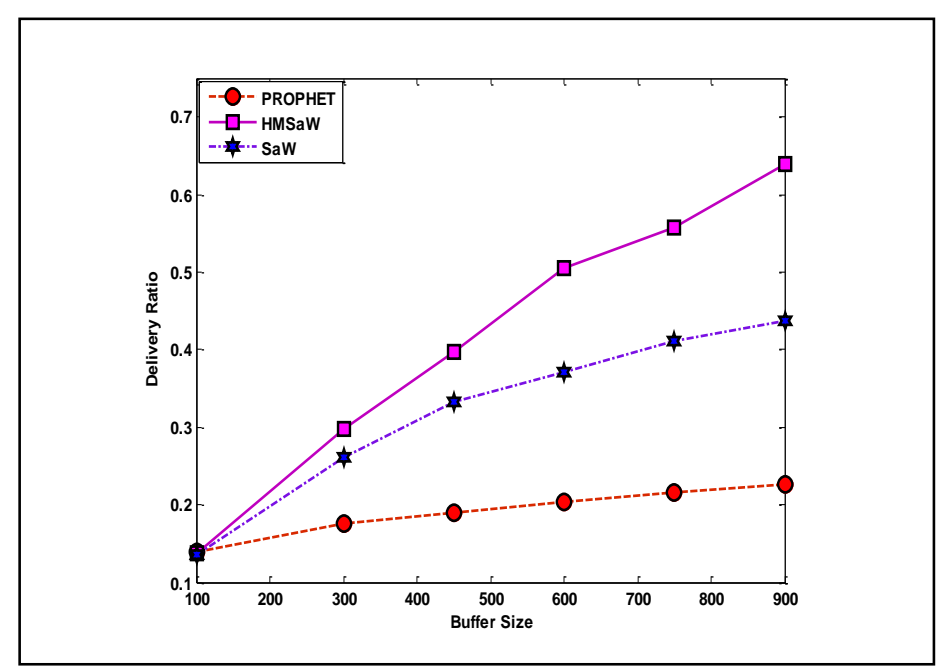

Figure 2. Impact of Varying Buffer Size on Delivery Ratio

As buffer size increases, more number of messages to be received towards destination as shown in Figure 2. It means larger queue size means more messages can be buffered, and risk of throwing away a message decreases. Among these routing protocols HMSaW outperforms in terms of delivery ratio when compared to Spray \& Wait and PROPHET routing protocols

Also by increases buffer size, the average delay experienced by Spray and Wait and HMSaW will be low compared to PROPHET routing protocols shown in Figure 3. 


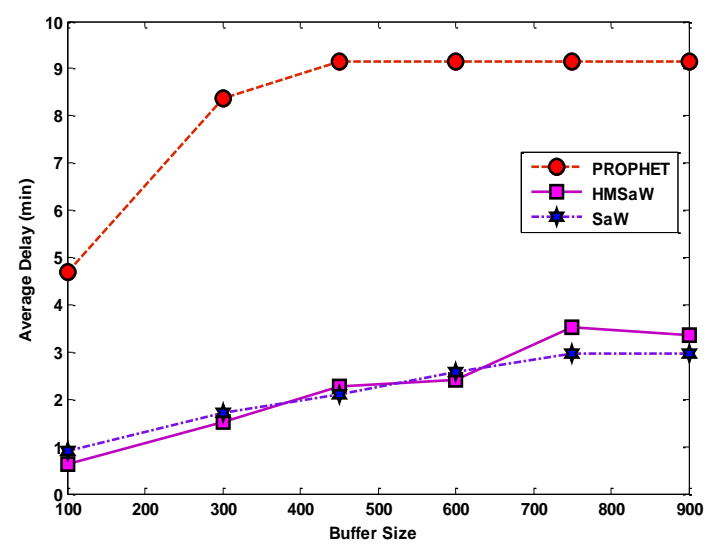

Figure 3. Impact of Varying Buffer Size on Average Delay

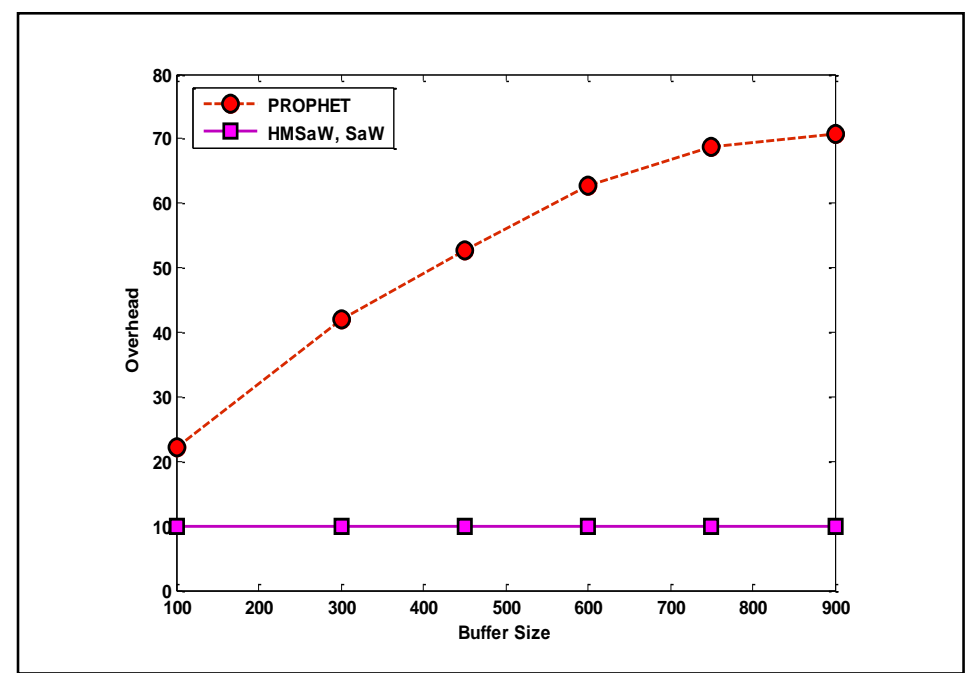

Figure 4. Impact of Varying Buffer Size on Overhead

As shown in Figure 4, the lowest transmission cost for delivering a packet in terms of network overhead is achieved by Spray and Wait and HMSaW. This is because there is a bounded on the total number of relay copies of a message called L copy. The overhead for PROPHET increases with increase in buffer size because more packets can be stored in the buffer and dropping of packets is reduced. Therefore, more packets are relayed through the network.

\subsubsection{Impact of Varying Number of Nodes (N)}

Increasing the number of network nodes, while fix the buffer size, improves the connectivity of the network nodes and allows for more packets to be delivered, means while increasing nodes, the delivery ratio also increases as shown in Figure 5. Along with these routing protocols, larger network size illustrates better performance for all protocols. In this scenario performance of HMSaW is better than PROPHET and SaW. 


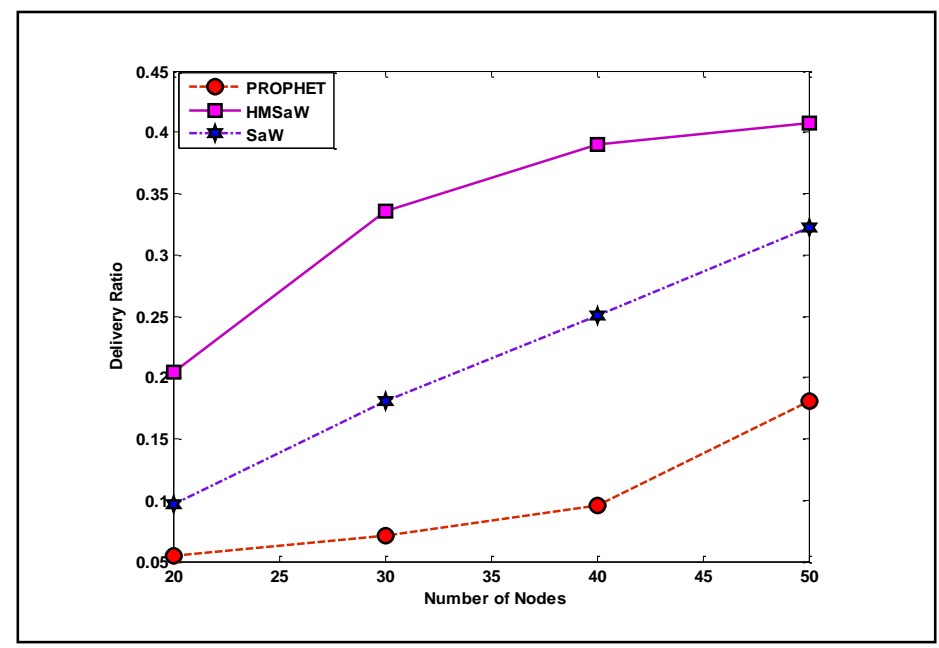

Figure 5. Impact Of Varying Number Of Nodes On Delivery Ratio

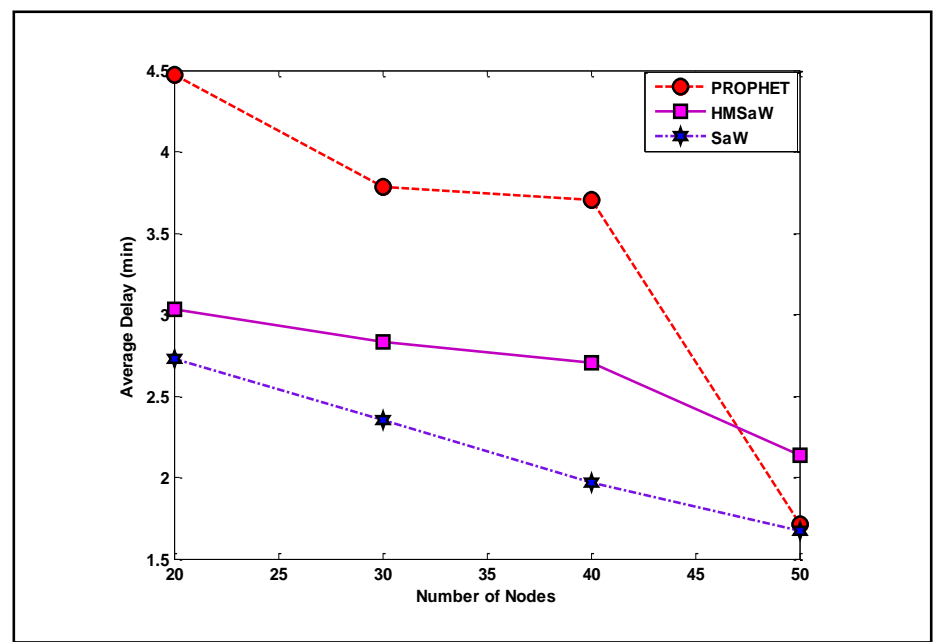

\section{Figure 6. Impact Of Varying Number Of Nodes On Average Delay}

It is observed from Figure 6 that average delay for all these routing protocol is constantly deceases with increasing network nodes from 20 to 50.The reason for that network nodes increases means opportunity to encounter between two node is also increases. The basis for that is the opportunity to encounter between any two nodes is increasing as the networks vary from sparse to dense. And also, because of using the "store-carry-forward" paradigm, it needs more relay nodes to store, more transmission and less time utilize to send packets to the destination as the number of the hops to the destination node increase. The Figure 6 represents that number of node size increases results in relay hops increases, and in conclusion average delay decreases. The average delay is constantly decreases, and the Spray and Wait routing protocol has the lowest network average delay. 


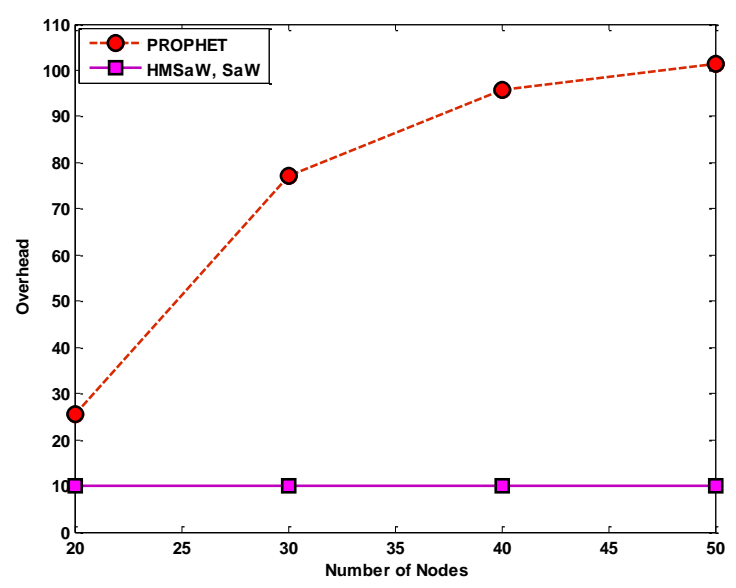

Figure 7. Impact of Varying Number of Nodes on Overhead

As shown in Figure 7, the lowest transmission cost for delivering a packet in terms of network overhead is achieved by Spray and Wait and HMSaW. This is because there is a bound on the total number of relay copies of a message called L copy. The overhead for PROPHET increases with increased number of network.

\subsubsection{Impact of Varying Transmission Range}

As the transmission range increases from 20 to 50 meter, more number of messages to be received towards destination as shown in Figure 8. It means increasing transmission range delivery ratio also increases. Among these routing protocols HMSaW outperforms in terms of delivery ratio when compared to Spray and Wait and PROPHET routing protocols.

Also by increases transmission range, the Figure 9 represents that, HMSaW with marginal reduction in average delay compared to existing PROPHET and Spray and Wait Routing protocol.

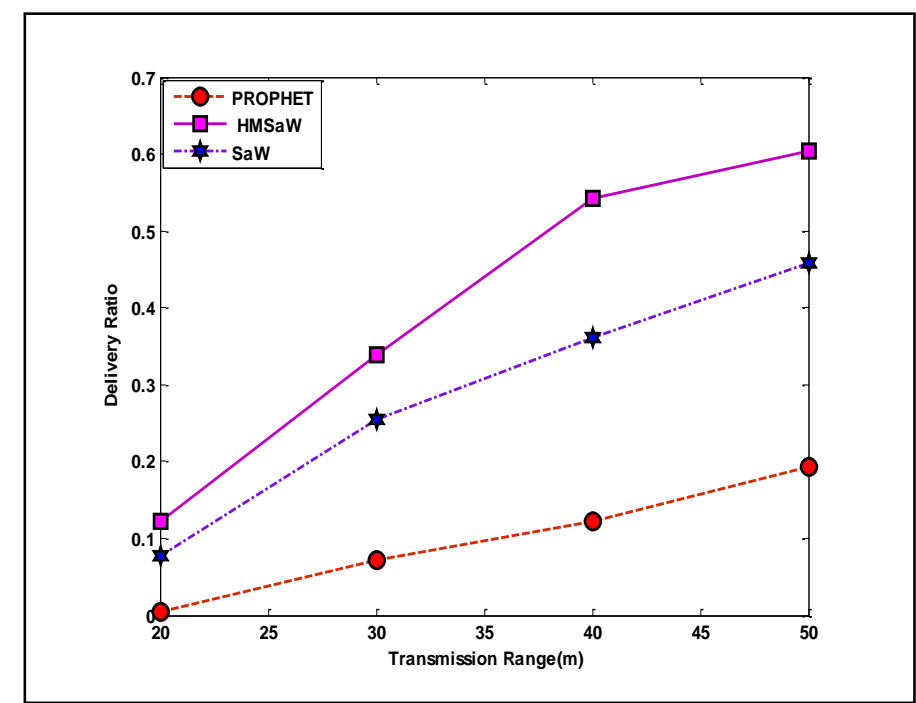

Figure 8. Impact of Varying Transmission Range on Delivery Ratio 


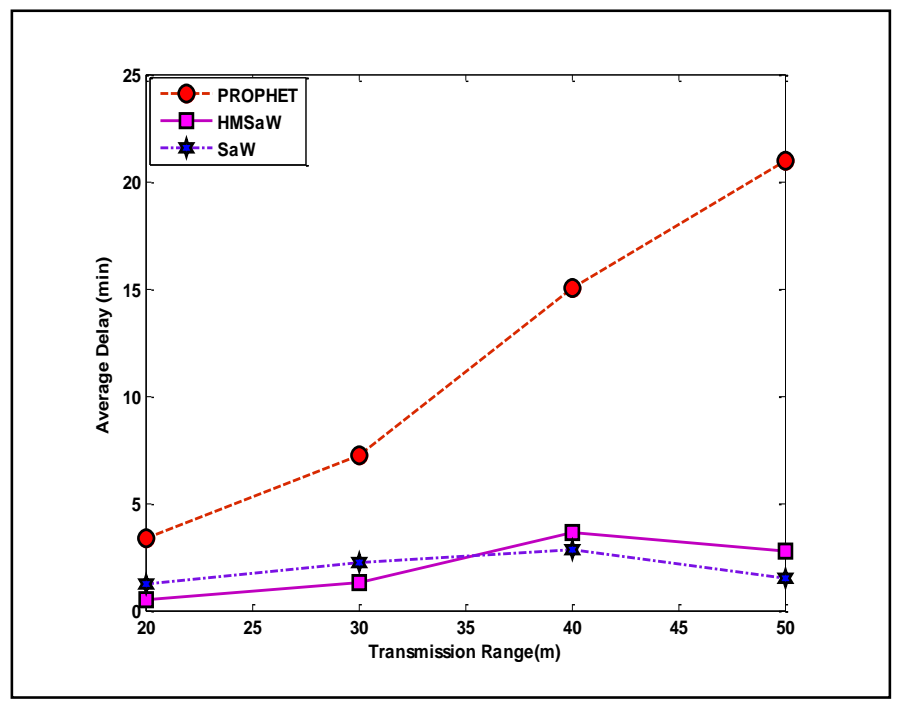

Figure 9. Impact of Varying Transmission Range on Average Delay

As shown in Figure 10, the lowest transmission cost for delivering a packet in terms of network overhead is achieved by Spray and Wait and HMSaW. The overhead for PROPHET increases with increases transmission range.

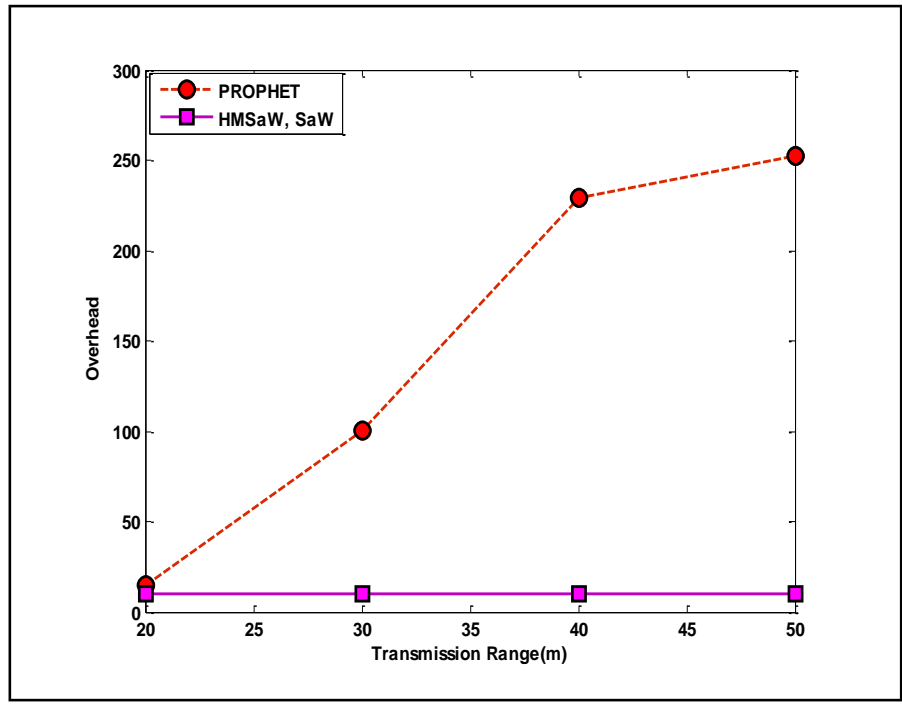

Figure 10. Impact of Varying Transmission Range on Overhead

\section{Conclusion}

This paper desribed an opportunistic novel routing algorithm. This routing protocol makes use of truncated power law distribution of pause time and flight length for human mobility. This performance of the novel algorithm's reflects super flight length and super pause time. HMSaW described to deliver the predefine number of copies to the destination during the spray phase based on delivery probability. In its wait phase,the remaining last single copy directly delivered to the destination. Proposed evaluation of HMSaW routing protocol based on MATLAB simulator in different scenarios. Simulation 
experiments show, proposed HMSaW outperforms the other routings in terms of the delivery ratio as compare with PROPHET and Spray \& Wait.

\section{References}

[1] S. Jain, K. Fall, and R. Patra, "Routing in a delay tolerant network," in ACM SIGCOMM'04, Oregon, Portland, (2004).

[2] [Online]. Available: http://www.dtnrg.org/wiki

[3] Cerf, V., Burleigh, S., Hooke, A., Torgerson, L., Durst, R., Scott, K., Fall, K., Weiss, H.: RFC 4838, Delay Tolerant Networking Architecture. IRTF DTN Research Group (2007)

[4] Amin Vahdat and David Becker, "Epidemic Routing for Partially-Connected Ad Hoc Networks," Technical Report CS-200006, Duke University, April (2000).

[5] A. Lindgren, A. Doria, and O. Schelen, "Probabilistic Routing in Intermittently Connected Networks", Mobile Computing and Communications Review, Vol. 7, No. 3, pp. 19 - 20, July, (2003).

[6] T. Spyropoulos, K. Psounis, and C. Raghavendra, "Spray and wait: an efficient routing scheme for intermittently connected mobile networks," in Proceedings of the ACM SIGCOMM Workshop on DelayTolerant Networking, pp. 252-259, ACM, (2005).

[7] I. Rhee, M. Shin, S. Hong, K. Lee, S. J. Kim, and S. Chong,"On the levy-walk nature of human mobility," IEEE/ACM Transactions on Networking, vol. 19, no. 3, pp. 630-643, (2011).

[8] M. B. Shah, S. N. Merchant And U. B. Desai, "Human Mobility Based Sensor Context Aware Routing protocol for Delay Tolerant Data Gathering in Multisink Cell-Phone Based Sensor Networks", International Journal of Distributed Sensor Network, Volume 2012, July (2012).

[9] [9] P. Hui, A. Chaintreau, J. Scott, " Pocket Switched Networks and Human Mobility in Conference Enviornment", Proceeding of the 2005 ACM workshop on Delay Tolerant Networking. ACM, (2005).

\section{Authors}

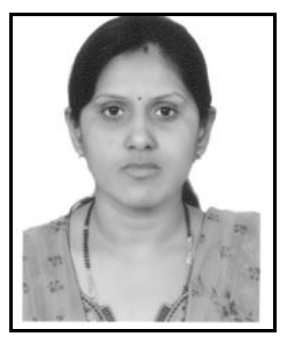

Namita Mehta, She is working as a Lecturer at the Department of Electronics \& Communication Engg, B \& B Institute of Technology, V.V.Nagar, Gujarat, India. I completed my B.E. in year 2005.My Master in Communication Engg. is completed from G.H.Patel College of Engg. \& Technology.

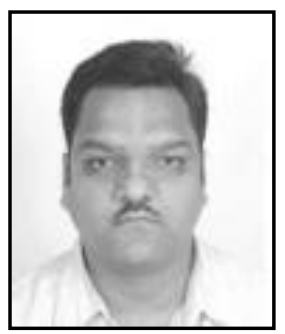

Mehul Shah, he is presently working as Assistant Professor in Electronics \& Communication Department, G.H.Patel College of Engg. \& Technology. He is currently completing a Ph.D. from IIT Bombay, Electrical Engineering Department, India. He worked on Cell phone based Wireless Sensor Networks and actively involved in Indo-UK Next generation network project on Pervasive sensor Environment. His research interests are in Wireless Communication and Mobile Adhoc \& Sensor Networks. 